\title{
Modelación matemática para el ajuste óptimo predictivo sobre el riesgo ecotoxicológico generado por el software multifuncional gecotoxic
}

Mathematics modelling for the optimum adjustment predictiv on the ecotoxicologic risk generated by the multifuntional software

gecotoxic asbtract

Recibido: marzo 11 de 2017 | Revisado: abril 10 de 2017 | Aceptado: mayo 15 de 2017

\author{
George Argota Pérez \\ Alberto Carbonell Marcé ${ }^{I}$ \\ CÉsar Portuondo Hernández ${ }^{1}$ \\ Jorge Lameriro Marín ${ }^{\mathrm{I}}$ \\ Miguel Rodríguez Alpízar ${ }^{\mathrm{I}}$ \\ Martín Merma Bellido ${ }^{2}$
}

$\overline{1 \text { Centro de }}$ Investigaciones Avanzadas y formación Superior en Educación, Salud y Medio Ambiente "AMTAWI". Puno - Perú. george.argota@gmail.com accarbonell1987@gmail.com cphmr87@yandex.com jlameiro87@yandex.com miguelalpizar@nauta.cu

2 Facultad Física-Matemática. Universidad Nacional del Altiplano (UNA). Puno - Perú. math_lid@hoymail.com

\begin{abstract}
RESUMEN
La modelación matemática aplicada a los diseños computacionales para predecir de forma exacta los impactos que se generan ambientalmente ha cobrado auge en los últimos años. El objetivo de esta investigación fue la modelación matemática para el ajuste óptimo predictivo del riesgo ecotoxicológico generado por el software multifuncional GECOTOXIC. La modelación fue acorde a los principios de la progresión aritmética donde se ensayaron para su optimización combinaciones numéricas asignadas a las variables de cada descriptor los cuales correspondieron al análisis residual de la carga contaminante como al efecto ambiental sobre el cuerpo de agua receptor. Las codificaciones consistieron en códigos arábigos, siendo analizada cada variable sobre datos reales de medición y cruzando sus interacciones de forma multifuncional para predecir el riesgo total, el cual se hizo mediante la comparación con daños ecotoxicológicos usando la especie Gambusia punctata como biomonitor. Los intervalos establecidos de ajuste óptimo para el riesgo de tipo bajo, medio y alto con relación a la similitud dada la magnitud del valor de riesgo correspondieron a: I), X-3X; II), 3X+1-5X; III), $5 \mathrm{X}+1-10 \mathrm{X}$, respectivamente. Se concluyó que fueron establecidos los rangos de intervalos para máximos y mínimos según progresión aritmética donde se asignaron, nuevos códigos arábigos de puntuación, ajustando el riesgo cualitativo según valores cuantitativos progresivos mediante análisis de similitud con daños en el biomonitor, en el que existe mayor precisión y exactitud en el riesgo que genera el nuevo software GECOTOXIC.
\end{abstract}

Palabras claves: modelación matemática, incertidumbrepredictiva, variablesmultifuncionales, probabilidad, riesgo eco toxicológico, software GECOTOXIC 


\begin{abstract}
Mathematical modeling applied to computer designs to predict accurately the environmental impacts generated, has boomed in recent years. The objective of this research was the mathematical modeling for the optimal predicive adjusment of the ecotoxicological risk generated by multifunctional software GECOTOXIC. The modeling was constructed in accordance with the principles of arithmetic progression, where numerical combinations assigned to the variables of each descriptor were tested for optimization, which corresponded to the residual analysis of the pollutant load as well as to the environmental effect on the receiving water body. The codifications consisted of Arabic codes, with each variable being analyzed on actual measurement data and cross-interacting its interactions in a multifunctional way to predict the total risk, which was achieved by comparing with Ecotoxicological damages using the species Gambusia punctata as bio monitor. The set intervals for optimal adjustment for the low, medium and high risk relative to the similarity given the magnitude of the risk value corresponded to: I), X-3X; II), 3X + 1-5X; III), 5X $+1-10 \mathrm{X}$, respectively. It was concluded that interval ranges for maxima and minima according to arithmetic progression were established where new Arabic scoring codes were assigned, adjusting the qualitative risk according to progressive quantitative values by means of analysis of similarity with damages in the biomonitor, where there is greater precision And accuracy in the risk generated by the new GECOTOXIC software.
\end{abstract}

Key words: mathematical modeling, predictive uncertainty, multifunctional variables, probability, ecotoxicological risk, software GECOTOXIC

\section{Introducción}

Existe un gran avance sobre los estudios que se refieren a la resolución de problemas (Schoenfeld, 2007) y donde se continúan las investigaciones desde diferentes enfoques. Debido a ello ha surgido un marcado interés por el análisis de los procesos de modelización matemática (Lesh \& Harel, 2003). Aunque no existe, una descripción uniforme sobre la modelación matemática (Cordero et. al, 2009) puede indicarse que la misma consiste en explicar el comportamiento de fenómenos en expresiones matemáticas a partir de problemas complejos, los que pueden demostrar conocimientos adquiridos en la presentación de supuestos o conjeturas (Trigueros, 2006).

Una forma de lograr la contextualización del conocimiento es la presentación de situaciones problemáticas reales que sean factibles de representarse mediante modelos matemáticos. Estos modelos aparecen cuando se tiene la necesidad de responder preguntas específicas, en situaciones reales como tomar decisiones o cuando es imperativo hacer prediccio- 
nes relacionadas con fenómenos naturales y sociales (Lehrer \& Schauble, 2000; Lesh \& English, 2005). El proceso de modelación se concibe como un todo y no como algo parcial (Camarena, 2000). Cualquier representación de la situación, a través de las matemáticas, se considera un modelo matemático (Barbosa, 2003, 2006).

Dentro de las grandes dificultades, en la actualidad, referida a la contaminación de las aguas por elementos tóxicos, es conocer cómo se expresan dichos elementos en el tiempo, relacionados con sus posibles efectos e impactos (Spahn \& Sherry, 1999). En ecotoxicología acuática, el uso de modelos cinético-matemáticos para predecir el comportamiento de elementos tóxicos, resulta de extraordinaria importancia, aunque generalmente, la aplicación de modelos cinético-matemáticos ha estado más relacionada con elementos xenobióticos y para ello, se ha requerido la utilización de conceptos sobre toxicocinética (Repetto \& Sanz, 1995).

Los estudios vinculados a softwares informáticos que puedan predecir el nivel de riesgo ecotoxicológico con su posible similitud, observada sobre organismos desarrollados en ambientes ecológicos naturales son limitados; sin embargo, uno de los realizados fue el de Argota \& Iannacone (2014), en que se presentó tal similitud entre el software GECOTOX y biomarcadores en la especie Gambusia punctata dada la carga contaminante residual, aun cuando puede valorarse como imprecisión por resolver, asegurar que los códigos asignados de mediación por parte del software, sigan siendo más precisos y exactos en la predicción comparativa, fundamentalmente, con cualquier magnitud del valor de riesgo que indican los biomarcadores en especies de tipo biomonitor.

El objetivo de la presente investigación fue la modelación matemática para el ajuste óptimo predictivo del riesgo ecotoxicológico, generado por el software multifuncional GECOTOXIC.

\section{Materiales y Métodos}

\section{Ámbito de estudio}

Se realizó un análisis exhaustivo y excluyente, a partir del software GECOTOX $^{\circ}$ (código de registro: 2027-2012, por el Centro Nacional de Derecho de Autor: CENDA) durante tres meses (octubre a diciembre del 2016). GECOTOX es operativo sobre la base de datos reales y no simulados donde la estimación de riesgo para el nuevo software GECOTOXIC se hizo mediante interacción matricial de números codificados, arábigamente, por intervalos de puntuación.

\section{Población y muestra}

Se estudió la base del funcionamiento predictivo del software GECOTOX, el que tuvo como variables de comprensión dos tipos de evaluaciones: análisis residual y efecto ambiental. Cada tipo de evaluaciones consideró varios menús que presentaron para su información de riesgo, un número arábigo asignado y específico.

Para la predicción del ajuste óptimo sobre la incertidumbre predictiva multifuncional se trabajó con mil doscientas variantes de estudio, que se 
seleccionaron atendiendo al carácter probabilístico aleatorio para evaluar sus posibles combinaciones y analizar el riesgo predictivo cualitativo de salida informacional (riesgo de tipo alto, medio, bajo).

\section{Descripción de las técnicas y procedimientos}

La técnica cuantitativa utilizada fue la de observación estructurada. El procedimiento a evaluar para el análisis residual presentó dos tipos de menús: 1) efecto toxicológico; y 2) tipo de tratamiento ambiental. En el primero, se refirió a tres sub-menús denominados: a) caracterización de la entidad, b) seguridad de parámetros físico-químicos y microbiológicos, así como, c) toxicidad del residual, mientras que en el segundo menú se incluyeron diversos tipos de análisis.

Para el caso del efecto ambiental, se consideraron cuatro tipos de menús: a) fuente contaminante, b) seguridad de parámetros físico-químicos, c) toxicidad del efecto ambiental y; d) análisis de indicadores biológicos.

Todas las variables fueron operacionalizadas sobre datos reales y no simulados, que realizaron los análisis de forma individual y luego, el riesgo fue analizado por los menús bajo efectos de interacción, los que se clasificaron como bajo, medio o alto. El principio de progresión aritmética considerado fue sobre la ecuación siguiente (Argota \& Iannacone, 2014):

- $A_{n}=A_{0}+r(n-1)$

Dónde:

- $\mathrm{A}_{\mathrm{n}}=\mathrm{n}$-ésimo término,

- $\mathrm{A}=$ valor inicial

- $r$ = razón

\section{Análisis estadístico}

Para el análisis estadístico, se consideró la probabilidad del riesgo como magnitud del valor de riesgo, que estuvo representado por las siguientes ecuaciones de cálculo:

- Probabilidad de riesgo
A. $[\mathrm{X}-3 \mathrm{X}]$ : bajo
B. $[3 X+1-5 X+4]$ : medio
C. $[5 \mathrm{X}+5-10 \mathrm{X}]$ : alto

- Magnitud del valor de riesgo
A. $[\mathrm{X}-3 \mathrm{X}]$ : trivial
B. $[3 X+1-5 X+4]$ : leve
C. $[5 X+5-10 X+5]$ : moderada
D. $[15 \mathrm{X}+1-15 \mathrm{X}+4]$ : severa
E. $[15 X+5-15 X+5]$ : extrema

\section{Resultados y Discusión}

Para la predicción de riesgo, generado de forma interactuada total, se siguieron los tres grupos de expresiones, según las progresiones aritméticas de la Tabla 1.

Tabla 1

Progresiones aritméticas secuenciales para optimizar el riesgo de predicción ambiental del software GECOTOX

\begin{tabular}{|rr|rr|rr|}
\hline$X$ & $3 X$ & $X$ & $X X$ & $3 X$ \\
$4 X$ & $7 X$ & $3 X+1$ & $6 X$ & $3 X+1$ & $5 X$ \\
$8 X$ & $11 X$ & $6 X+1$ & $9 X$ & $5 X+1$ & $10 X$ \\
\hline
\end{tabular}


Las expresiones presentadas constituyen una parte fundamental de la programación informática, ya que sirven para realizar una o varias operaciones sobre un dato o un conjunto de datos, obteniéndose luego, otro dato como resultado de interés. Los operadores definen algunas de las instrucciones que pueden realizarse dentro de una expresión como combinación de operadores y operandos. Los datos $\mathrm{u}$ operandos pueden ser constantes, variables y llamadas a funciones. Dentro de una expresión pueden encontrarse además, sub-expresiones encerradas entre paréntesis.

Cuando se ejecuta una sentencia de código que contiene una expresión, posteriormente, se evalúa la expresión, refiriendo cualquier valor que dependerá del asignado previamente a las variables, las constantes, operadores y funciones utilizadas, así como la secuencia de la ejecución dada las operaciones correspondientes. El valor resultante de la evaluación sobre la expresión, corresponderá a un determinado tipo de dato de manera que, relacionando las expresiones y tabulando valores para la variable $X$, se pueden obtener los intervalos de riesgos que se observan en la Tabla 2.

Tabla 2

Progresiones aritméticas secuenciales de grupo I

\begin{tabular}{|rr|}
\hline I) $X$ & $3 X(\mathrm{R} 1)$ \\
II) $4 X$ & $7 X(R 2)$ \\
III) $8 X$ & $11 X(R 3)$ \\
\hline
\end{tabular}

Si $\mathrm{X}$ es el valor de peligro $(\mathrm{X}=\mathrm{P}) \mathrm{y}$ esta se considera con un valor cero $(0)$, los valores de la expresión precedente y teniendo en consideración la ecuación de riesgo se presentarán las siguientes expresiones:

- $\mathrm{R}=0$ : no existe riesgo alguno en las tres expresiones.

Si $\mathrm{X}=\mathrm{P}=1$ y $\mathrm{V}=3 \mathrm{X}$

- $\mathrm{R}_{1}=1+3(1)=4$ este valor indica que existe un riesgo.

Si $\mathrm{P}=4 \mathrm{X}$ y $\mathrm{V}=7 \mathrm{X}$

- $\mathrm{R}_{2}=4(1)+7(1)=11$. Este valor indica que se incrementa el riesgo en un $175 \%$ respecto al valor del $R_{1}$ Si $\mathrm{P}=8 \mathrm{X}$ y $\mathrm{V}=11 \mathrm{X}$

- $\mathrm{R}_{3}=8(1)+11(1)=19$ este valor indica que se incrementa el riesgo en un $375 \%$ respecto al valor de $R_{1}$.

Asimismo, si el valor de $\mathrm{X}$ se tabula con valores positivos, el nivel de riesgo se incrementa donde al relacionar la siguiente expresión y tabulando valores para la variable $\mathrm{X}$, igualmente pueden obtenerse otros intervalos de riesgo como se observa en la Tabla 3.

Tabla 3

Progresiones aritméticas secuenciales de grupo II

\begin{tabular}{|rr|}
\hline I) $X$ & $3 X$ (R1) \\
II) $3 X+1$ & $6 X(R 2)$ \\
III) $6 X+1$ & $9 X(R 3)$ \\
\hline
\end{tabular}

Realizando el análisis para las progresiones aritméticas secuenciales del grupo II, puede mencionarse que, si $\mathrm{X}$ es el valor de peligro $(\mathrm{X}=\mathrm{P})$ y esta se considera con un valor cero $(0)$, los valores de la expresión precedente y teniendo en consideración la ecuación de riesgo, entonces en otro tipo de análisis, podrán presentarse las siguientes expresiones: 
Si $\mathrm{X}=\mathrm{P}=0$ y $\mathrm{V}=3 \mathrm{X}$

- $\mathrm{R}_{1}=0 ;$ No existe riesgo

- $\mathrm{R}_{2}=3(0)+1+6(0)=1 ;$ sí, existe riesgo

- $\mathrm{R}_{3}=6(0)+1+9(0)=1$; sí, existe riesgo Si $\mathrm{X}=1, \mathrm{P}=3 \mathrm{X}+1$ y $\mathrm{V}=6 \mathrm{X}$

- $\mathrm{R}_{1}=1+3(1)=4$; sí, existe riesgo

- $\mathrm{R}_{2}=3(1)+1+6(1)=10$; este valor indica que existe un riesgo de $150 \%$ respecto a $R_{1}$

- $\mathrm{R}_{3}=6(1)+1+9(1)=16$; este valor indica que existe un riesgo de 300\% respecto a $R_{1}$

Finalmente, del análisis para las progresiones aritméticas secuenciales del grupo III, de forma similar se puede mencionar que, si $\mathrm{X}$ es el valor de peligro $(\mathrm{X}=\mathrm{P})$ y este se considera con un valor cero (0), los valores de la expresión precedente y teniendo en consideración la ecuación de riesgo, entonces este tipo de análisis podrá presentarse según las siguientes expresiones y obtenerse los intervalos de riesgos que se muestran en la Tabla 4.

Tabla 4

Progresiones aritméticas secuenciales de grupo III

\begin{tabular}{|rc|}
\hline I) $X$ & $3 X$ (R1) \\
II) $3 X+1$ & $5 X(R 2)$ \\
III) $5 X+1$ & $10 X(R 3)$ \\
\hline
\end{tabular}

Si $\mathrm{X}=\mathrm{P}=0$ y $\mathrm{V}=3 \mathrm{X}$

- $\mathrm{R}_{1}=0 \quad$ No existe riesgo

- $\mathrm{R}_{2}=3(0)+1+5(0)=1 \quad$ Sí, existe riesgo.

- $\mathrm{R}_{3}=5(0)+1+10(0)=1 \quad$ Sí, existe riesgo.
Si $X=1, P=3 X+1$ y $V=5 X$

- $\mathrm{R}_{1}=1+3(1)=4 \quad$ Sí, existe riesgo.

- $\mathrm{R}_{2}=3(1)+1+5(1)=9$. Este valor indica que existe un riesgo de $125 \%$ respecto a $R_{1}$

- $\mathrm{R}_{3}=5(1)+1+10(1)=16$. Este valor indica que existe un riesgo de $300 \%$ respecto a $R_{1}$

Si el valor de $\mathrm{X}$ se tabula con valores positivos, el nivel de riesgo, igualmente se incrementa.

Para la asignación del código arábigo, se realizó, previamente, la descripción del efecto ambiental donde el software fue dependiente de tres condiciones iniciales: fuente contaminante, seguridad de parámetros físico-químicos y análisis de indicadores biológicos naturales. En cuanto a la fuente contaminante, la misma consideró una zona de análisis (aproximación, mezcla y exposición), así como el número de fuentes identificadas y los contaminantes de interés (que se expresan por matrices ambientales). De igual manera, fue considerada la seguridad de parámetros físicoquímicos y microbiológicos (cantidad de parámetros medidos como el número de ellos encontrados, según la norma utilizada), así como la toxicidad del efecto ambiental sobre la base del tipo a evaluar (sustancia, compuesto o mezcla), el ensayo (microcosmo o batería) y el número de pruebas que presentan toxicidades, siendo las mismas impares (3 o 5) a determinar (Tabla 5). 
Tabla 5

Descripción del efecto ambiental generado por el software GECOTOX

\begin{tabular}{|c|c|c|c|c|c|c|c|c|}
\hline \multicolumn{5}{|c|}{ FUENTE CONTAMINANTE } & \multicolumn{4}{|c|}{$\begin{array}{l}\text { SEGURIDAD DE PARÁMETROS } \\
\text { FÍSICO-QUÍMICOS }\end{array}$} \\
\hline \multicolumn{5}{|c|}{ Zona } & \multirow{3}{*}{$\begin{array}{l}\text { Número de } \\
\text { parámetros }\end{array}$} & \multirow{3}{*}{ Categ. } & I & 4 \\
\hline 1 & aproximación & \multicolumn{3}{|c|}{$\begin{array}{l}\text { Contaminantes de } \\
\text { interés }\end{array}$} & & & II & 2 \\
\hline 2 & mezcla & I & II & $\geq 3$ & & & III & 0 \\
\hline 3 & exposición & 1 & 2 & 3 & \multirow{2}{*}{$\begin{array}{c}\text { Cantidad de } \\
\text { parámetros válidos }\end{array}$} & \multicolumn{2}{|c|}{ cumplen } & 0 \\
\hline \multicolumn{5}{|c|}{$\begin{array}{l}\text { ANÁLISIS EN INDICADORES BIOLÓGICOS } \\
\text { NATURALES }\end{array}$} & & \multicolumn{2}{|c|}{$\begin{array}{l}\text { No } \\
\text { cumplen }\end{array}$} & 1 \\
\hline \multicolumn{5}{|c|}{ Daños } & & & & \\
\hline & Sin daños & \multicolumn{3}{|c|}{1} & & & & \\
\hline & Ligero & \multicolumn{3}{|c|}{2} & & & & \\
\hline & Leve & \multicolumn{3}{|c|}{3} & & & & \\
\hline & Moderado & \multicolumn{3}{|c|}{4} & & & & \\
\hline & Extremo & \multicolumn{3}{|c|}{5} & & & & \\
\hline
\end{tabular}

Finalmente, el análisis de indicadores biológicos consideró el tipo de muestra, la cual fue evaluada atendiendo a la magnitud de daño, siguiendo la clasificación reportada por Argota \& Iannacone (2014).

Fue necesario evaluar las cargas contaminantes de residuales ambientales, tributados por las fuentes identificadas como contaminantes o potenciales contaminantes donde se concibió, una caracterización inicial sobre el efecto toxicológico que efectúan las mismas y que para ello, fueron considerados como menús el análisis de riesgo residual, seguridad de parámetros físico-químicos y toxicidad del efluente (Tabla 6).

Tabla 6

Efecto toxicológico generado por el software GECOTOX.

\begin{tabular}{|c|c|c|c|c|c|c|c|c|c|c|}
\hline \multicolumn{7}{|c|}{ ANÁLISIS DE RIESCO RESIDUAL } & \multicolumn{4}{|c|}{$\begin{array}{c}\text { SEGURIDAD DE } \\
\text { PARAMETROS FÍSICO- } \\
\text { QUIMICOS }\end{array}$} \\
\hline & & & & & & & \multirow{3}{*}{$\begin{array}{l}\text { Número de } \\
\text { parámetros }\end{array}$} & \multirow{3}{*}{ Categ. } & I & 5 \\
\hline \multirow{4}{*}{$\begin{array}{l}\text { Tipo do } \\
\text { fuente }\end{array}$} & \multicolumn{2}{|c|}{ difusa } & \multirow{4}{*}{\multicolumn{2}{|c|}{$\begin{array}{l}\text { Tipo de } \\
\text { emisión }\end{array}$}} & $\begin{array}{c}\text { Continua de } \\
\text { larga } \\
\text { duración }\end{array}$ & \multirow[b]{2}{*}{3} & & & II & 3 \\
\hline & \multicolumn{2}{|r|}{2} & & & $\begin{array}{l}\text { Continua de } \\
\text { corta } \\
\text { duración }\end{array}$ & & & & III & 1 \\
\hline & \multicolumn{2}{|c|}{ puntual } & & & $\begin{array}{l}\text { Intermitente } \\
\text { de alta } \\
\text { frecuencia }\end{array}$ & 4 & \multirow{2}{*}{$\begin{array}{l}\text { Cantidad de } \\
\text { parámetros } \\
\text { válidos }\end{array}$} & \multicolumn{2}{|c|}{ cumplen } & 0 \\
\hline & \multicolumn{2}{|r|}{1} & & & $\begin{array}{l}\text { Intermitente } \\
\text { de baja } \\
\text { frecuencia }\end{array}$ & 2 & & \multicolumn{2}{|c|}{$\begin{array}{c}\text { No } \\
\text { cumplen }\end{array}$} & 1 \\
\hline \multicolumn{7}{|c|}{ Toxicidad del efluente } & & & & \\
\hline \multirow[t]{3}{*}{ mezcla } & tóxica & 1 & \multicolumn{2}{|c|}{$\begin{array}{c}\text { Bio } \\
\text { degradabilidad }\end{array}$} & \multicolumn{2}{|c|}{$\begin{array}{c}\text { Caracterización del } \\
\text { nesgo }\end{array}$} & & & & \\
\hline & \multirow[t]{2}{*}{$\begin{array}{c}\text { No } \\
\text { tôxica }\end{array}$} & \multirow[t]{2}{*}{0} & \multicolumn{2}{|l|}{$\leq 50 \%$} & $\begin{array}{l}\text { PEC }> \\
\text { PNEC }\end{array}$ & 1 & & & & \\
\hline & & & $>50 \%$ & 3 & $\begin{array}{l}\text { PEC }< \\
\text { PNEC }\end{array}$ & 2 & & & & \\
\hline
\end{tabular}


Una vez asignados los códigos de puntuación para cada variable por menús que correspondieron a los descriptores se procedió a su lenguaje de programación, así como a las evaluaciones de situaciones ambientales (Figuras 1 y 2, respectivamente).
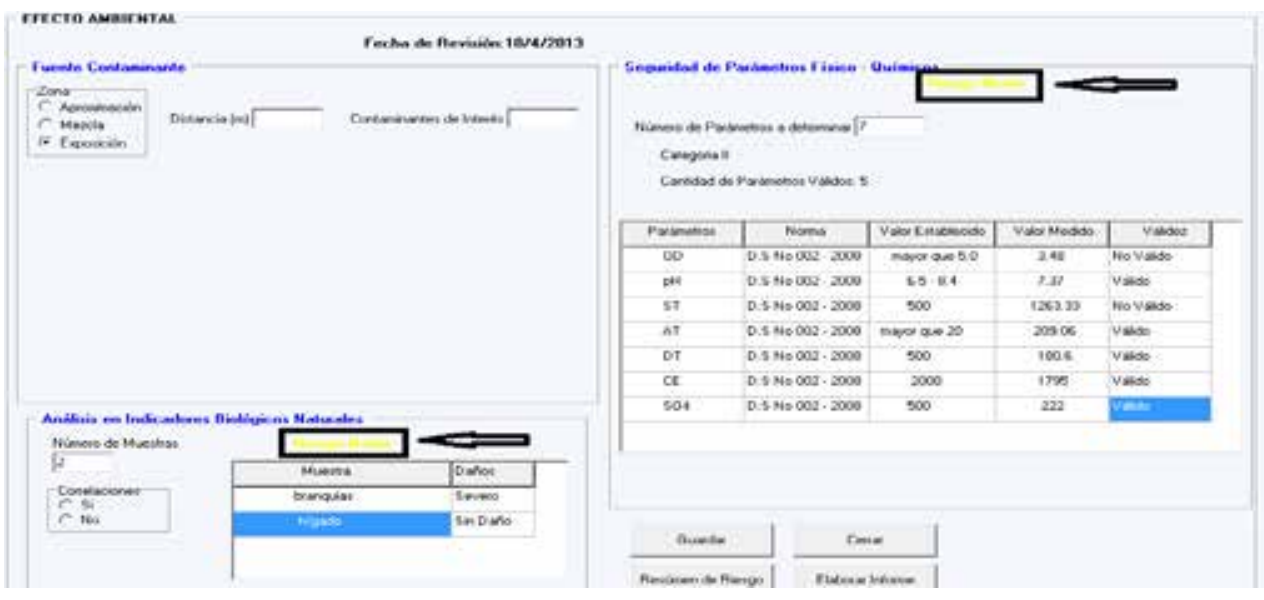

Figura 1. Descriptor del efecto ambiental generado por el software GECOTOX

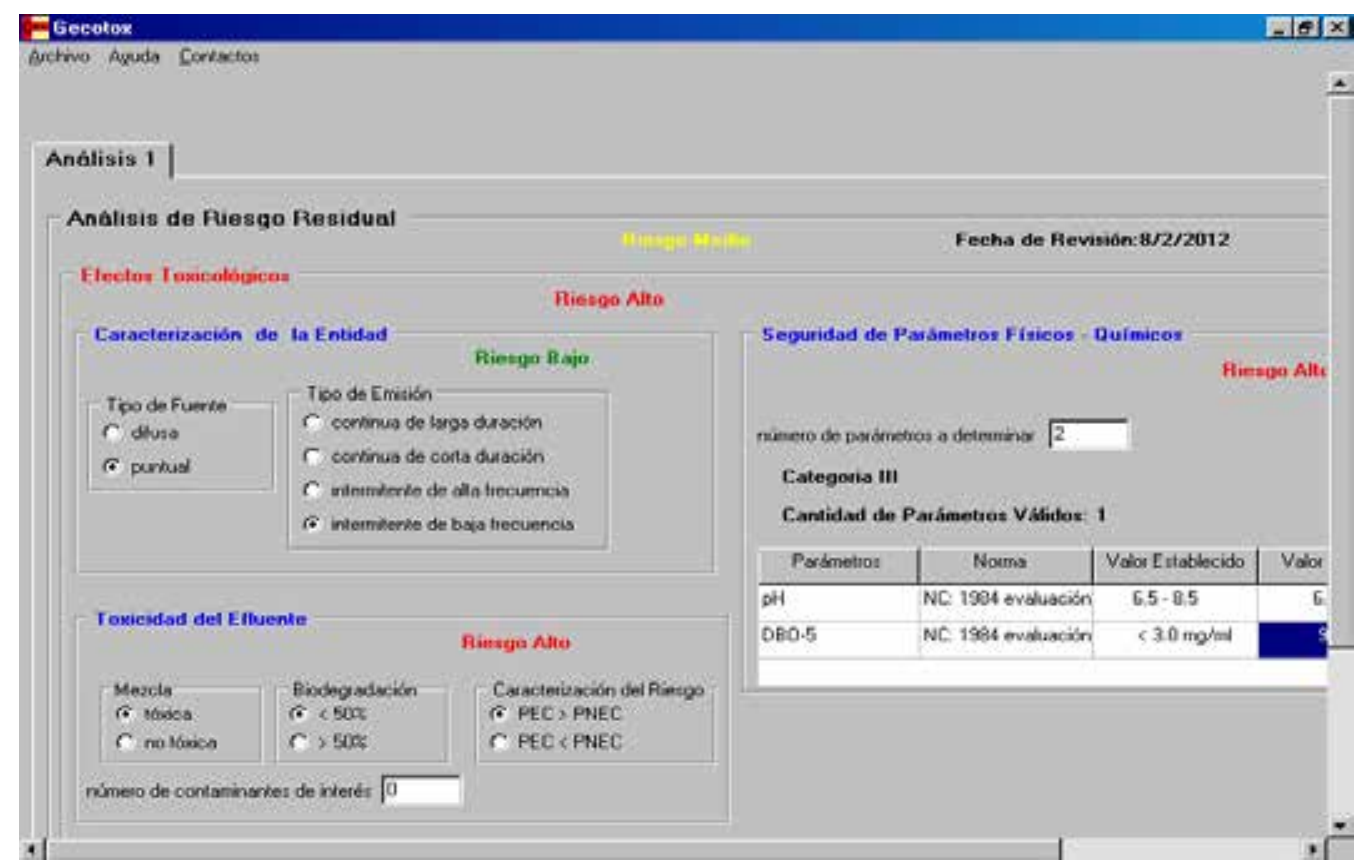

Figura 2. Descriptor del análisis de riesgo residual generado por el software GECOTOX

Cruzando el concepto de rangos con los temas de tratamiento ambiental se encontraron, intervalos, en la fase de análisis de riesgo del protocolo de evaluación de riesgo ecológico mediante la magnitud del valor de riesgo indicado por Argota \& Iannacone (2014) como se observa en la Tabla 7. 
Tabla 7

Magnitud del valor de riesgo

\begin{tabular}{ccccc}
\hline \multirow{2}{*}{ MVR } & & \multicolumn{3}{c}{ Consecuencias } \\
& & baja & media & alta \\
\hline \multirow{2}{*}{ Probabilidad } & baja & trivial & leve & moderada \\
& media & leve & moderada & severa \\
& alta & moderada & severa & extrema \\
\hline
\end{tabular}

Fuente: Argota \& Iannacone, 2014.

Al considerarse la Tabla 7 , pudo obtenerse tres tipos de probabilidad de riesgo, tres tipos de consecuencias y nueve magnitudes del valor del riesgo, así

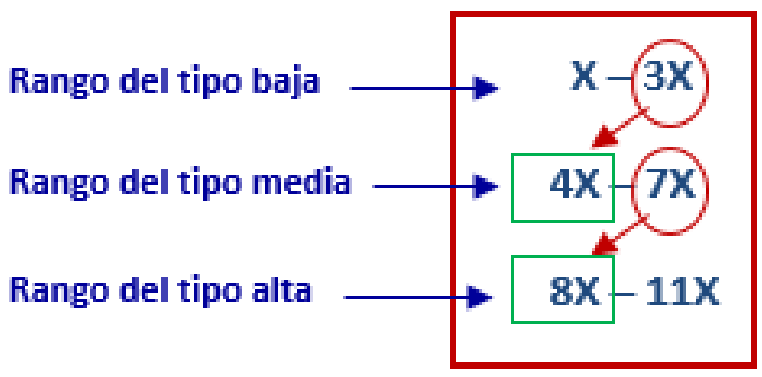

como grupos de tres rangos de los tipos de consecuencias que corresponden a rangos de riesgos por grupos de intervalos como se muestran en la Figura 6.
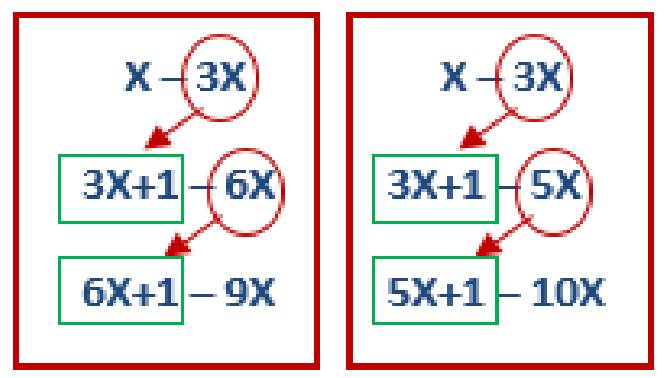

Figura 6. Correspondencia de rangos de riesgos por grupos de intervalos

Conjuntamente, se tienen tres grupos de intervalos con diferentes secuencias de ecuaciones donde sí se considera que, en la elaboración de los intervalos, se razonan las progresiones aritméticas de cada caso, pueden interpretarse sobre la presencia de tres casos y que cada uno presenta su propia progresión aritmética (Figura 7).

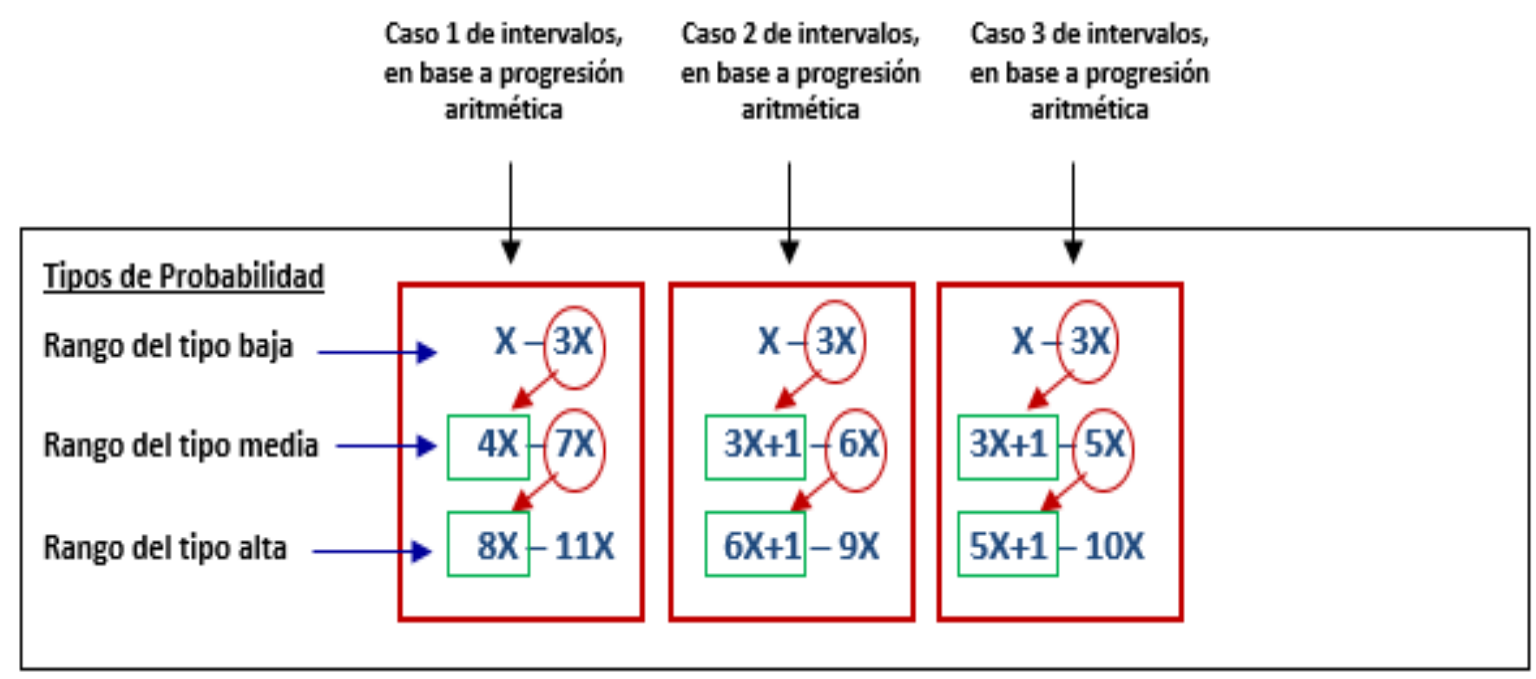

Figura 7. Progresión aritmética por intervalos y rangos de riesgo según códigos redesignados 
Realizando un ensayo y considerando $\mathrm{X}=1$, entonces se tiene una primera matriz de datos que describe el tipo de rango en correspondencia con la probabilidad de daño o consecuencia (Figura 8).

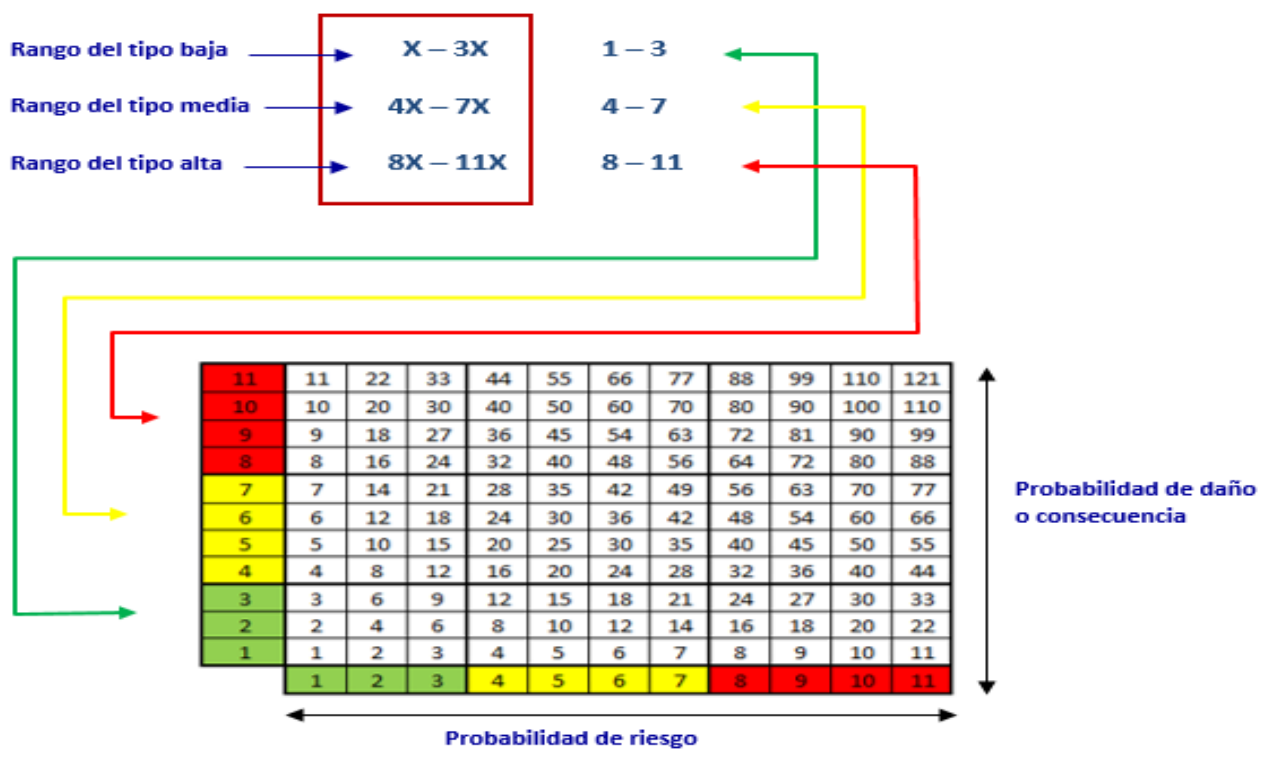

Figura 8. Matriz de interacción entre probabilidad de daño o consecuencia $\mathrm{y}$ rangos por intervalos de riesgo

Integrando el cuadro de la magnitud del valor del riesgo, multiplicando la probabilidad del riesgo por la probabilidad de la consecuencia o dańo, pueden obtenerse, los nueve grupos de magnitudes del valor de riesgo, lo cual coincide con lo indicado por la Tabla 6 .
Si evalúa, además, la interpretación de la Tabla 6, puede obtenerse la comparación, lo cual es coincidente y por tanto, puede dimensionarse su ajuste óptimo para la predicción de riesgo ecotoxicológico ambiental como se muestra en la Figura 9.

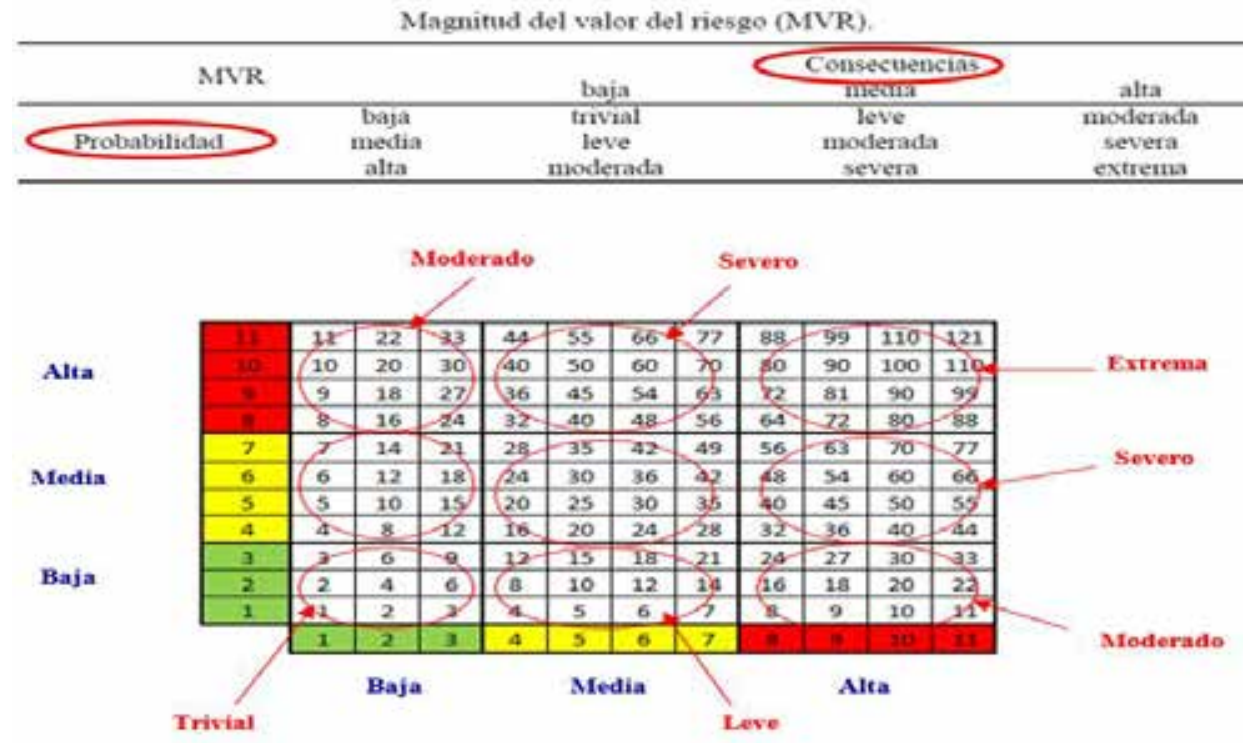

Figura 9. Matriz de ajuste óptimo de predicción para la interacción entre probabilidad de daño o consecuencia y rangos por intervalos de riesgo 
Sin embargo, si bien fue cierto que se tuvo la matriz de ajuste, esto no significa que existió correspondencia en cuanto a la predicción de riesgo que se generaba por el software con la similitud de daños valorada en situaciones reales como puede mostrase en la Tabla 8.

Tabla 8

Similitud de predicción de riesgo estimado por exposición a metales pesados

\begin{tabular}{|c|c|c|c|c|}
\hline \multirow{2}{*}{ Herramienta } & \multicolumn{4}{|c|}{ Magnitud del valor de riesgo } \\
\hline & \multicolumn{2}{|c|}{ Análisis I } & \multicolumn{2}{|c|}{ Análisis II } \\
\hline GECOTOX $^{\circledR}$ & \multicolumn{2}{|c|}{ Medio* } & \multicolumn{2}{|c|}{ Medio* } \\
\hline \multirow{2}{*}{$\begin{array}{l}\text { Especie Gambusia } \\
\text { punctata }\end{array}$} & \multicolumn{2}{|c|}{ Alto ** } & \multicolumn{2}{|c|}{ Medio ** } \\
\hline & $\mathrm{Cu}$ & $44.08 \pm 2.11$ & $\mathrm{Cu}$ & $32.27 \pm 3.42$ \\
\hline
\end{tabular}

Leyenda: medio *: considerando el cuadro 4; alto ** considerando análisis de histopatología en branquias (Argota et al., 2012).

En la Tabla 9, puede mostrarse la comparación entre los valores de $\mathrm{Cu}$ bioacumulado en branquias entre dos análisis realizados para la misma especie, mismo lugar y en dos momentos diferentes con relación a la utilización de GECOTOX donde las condiciones de evaluación fueron similares.

Tabla 9

Resumen estadistico

\begin{tabular}{|l|c|c|}
\cline { 2 - 3 } \multicolumn{1}{c|}{} & $\mathrm{Gp} \mathrm{Cu} \mathrm{1}$ & $\mathrm{Gp} \mathrm{Cu} \mathrm{2}$ \\
\hline Recuento & 3 & 3 \\
\hline Promedio & 44.08 & 32.27 \\
\hline Desviación estándar & 0.01 & 0.01 \\
\hline $\begin{array}{l}\text { Coeficiente de } \\
\text { variación }\end{array}$ & $0.02 \%$ & $0.03 \%$ \\
\hline Mínimo & 44.07 & 32.26 \\
\hline Máximo & 44.09 & 32.28 \\
\hline Rango & 0.02 & 0.02 \\
\hline Sesgo estandarizado & $-2.26 \mathrm{E}-12$ & $-2.26 \mathrm{E}-12$ \\
\hline
\end{tabular}

Esta tabla contiene el resumen estadístico para las dos muestras de datos. De particular interés es el sesgo estandarizado que puede usarse para comparar si las muestras provienen de distribuciones normales. Valores de estos estadísticos fuera del rango de $-2 \mathrm{a}+2$ indican desviaciones significativas de la normalidad, lo que tendería a invalidar las pruebas que comparan las desviaciones estándar. En este caso, ambos valores de sesgo estandarizado se encuentran dentro 
del rango esperado. En cuanto a la comparación de las medias, entre ambos análisis, pudo obtenerse lo siguiente:

\section{Comparación de medias}

- Intervalos de confianza del $95.0 \%$ para la media de Gp Cu 1: $44.08+/-$ 0.03 [44.0552, 44.11]

- Intervalos de confianza del $95.0 \%$ para la media de Gp Cu 2: 32.27 +/0.03 [32.2452, 32.30]

- Intervalos de confianza del $95.0 \%$ intervalo de confianza para la diferencia de medias

- Suponiendo varianzas iguales: 11.81 +/- $0.02[11.79,11.84]$

\section{Prueba t para comparar medias}

- Hipótesis nula: media1 = media2

- Hipótesis Alt.: media1 <> media2

- Suponiendo varianzas iguales: $\mathrm{t}=$ 1446.42 valor-P $=1.37 \mathrm{E}-12$

- Se rechaza la hipótesis nula para alfa $=0.05$.

De interés particular fue el intervalo de confianza para la diferencia entre las medias, el cual se extendió desde 11.79 hasta 11.84. Puesto que el intervalo no contiene el valor 0 , existió diferencia, estadísticamente significativa, entre las medias de las dos muestras con un nivel de confianza del $95.0 \%$, lo cual explicó, que no era posible esperar cuando las condiciones ambientales eran similares, de manera que ello indicó la necesidad de realizar ajustes en cuanto a la asignación de puntos sobre las variables para el software, ya que el riesgo siguió informando la misma predicción. Para ello, fue necesario solo rediseñar los puntos asignados al menú que se refería a la seguridad de parámetros físicoquímicos, ya que al parecer indicaban intervalos muy abiertos en cuanto a la amplitud y por ende, su puntuación se encontraba por encima de lo real, siendo menos preciso y exacto el riesgo final por interacción (Tabla 10).

Asimismo, fue considerado entender el número de contaminantes de interés determinado(s) por matriz, ya que ello de igual modo, influye en los análisis de daño sobre los órganos o tejidos de los organismos que habitan en el ecosistema, objeto de estudio, estableciendo códigos de puntuación para los mismos como se observa en la Tabla 11.

Tabla 10

Reajuste de códigos de puntuación (derecha) / seguridad de parámetros fisico-quimicos

\begin{tabular}{|c|c|c|c|}
\hline \multicolumn{3}{|c|}{$\begin{array}{c}\text { SEGURIDAD DE PARÁMETROS } \\
\text { FISICO-QUIMICOS }\end{array}$} \\
\hline \multirow{2}{*}{$\begin{array}{c}\text { Número de } \\
\text { parámetros }\end{array}$} & Categ & I & 10 \\
\cline { 3 - 4 } & & II & 5 \\
\cline { 2 - 4 } & III & 3 \\
\hline $\begin{array}{c}\text { Cantidad de } \\
\text { parámetros válidos }\end{array}$ & cumplen & 0 \\
\cline { 2 - 4 } & $\begin{array}{c}\text { No } \\
\text { cumplen }\end{array}$ & 1 \\
\hline
\end{tabular}

\begin{tabular}{|c|c|c|c|}
\hline \multicolumn{4}{|c|}{$\begin{array}{c}\text { SEGURIDAD DE PARÁMITROS } \\
\text { FISICO-QUiMICOS }\end{array}$} \\
\hline \multirow{2}{*}{$\begin{array}{c}\text { Número de } \\
\text { parámetros }\end{array}$} & Categ & I & 5 \\
\cline { 3 - 4 } & II & 3 \\
\cline { 2 - 4 } & III & 1 \\
\hline $\begin{array}{c}\text { Cantidad de } \\
\text { parámetros válidos }\end{array}$ & cumplen & 0 \\
\cline { 2 - 4 } & $\begin{array}{c}\text { No } \\
\text { cumplen }\end{array}$ & 1 \\
\hline
\end{tabular}


Tabla 11

Código de la variable contaminante de interés

\begin{tabular}{|c|c|c|c|c|}
\hline \multicolumn{5}{|c|}{ FUENTE CONTAMINANTE } \\
\hline \multicolumn{5}{|c|}{ Zona } \\
\hline 1 & aproximación & \multicolumn{3}{|c|}{$\begin{array}{c}\text { Contaminantes de } \\
\text { interés }\end{array}$} \\
\hline 2 & mezcla & 1 & II & $\geq 3$ \\
\hline 3 & exposición & 1 & 3 & 5 \\
\hline
\end{tabular}

En la Tabla 12, se muestran las codificaciones finales para el menú referido al efecto ambiental que permitió la similitud del riesgo para un nuevo software (Figura 10), denominado GECOTOXIC con los daños analizados, independientemente, de cualquier condición que sea determinada en el medio.

El nuevo software no solo es más amigable en cuanto a sus ventanas de interacción, sino que incorporan nuevas

Tabla12

Código de puntuación del efecto ambiental ajustado

\begin{tabular}{|c|c|c|c|c|c|c|c|c|}
\hline \multicolumn{5}{|c|}{ FUENTE CONTAMINANTE } & \multicolumn{4}{|c|}{$\begin{array}{l}\text { SEGURIDAD DE PARÁMIETROS } \\
\text { FISICO-QUIMICOS }\end{array}$} \\
\hline \multicolumn{5}{|c|}{ Zona } & \multirow{3}{*}{$\begin{array}{l}\text { Número de } \\
\text { parámetros }\end{array}$} & \multirow{3}{*}{ Categ } & I & 5 \\
\hline 1 & aproximación & \multicolumn{3}{|c|}{$\begin{array}{l}\text { Contaminantes de } \\
\text { interés }\end{array}$} & & & II & 3 \\
\hline 2 & mezcla & I & II & $\geq 3$ & & & III & 1 \\
\hline 3 & exposición & 1 & 3 & 5 & \multirow{2}{*}{$\begin{array}{l}\text { Cantidad de } \\
\text { parámetros válidos }\end{array}$} & \multicolumn{2}{|c|}{ cumplen } & 0 \\
\hline \multicolumn{5}{|c|}{$\begin{array}{l}\text { ANÁLISIS EN INDICADORES } \\
\text { BIOLÓGICOS NATURALES }\end{array}$} & & \multicolumn{2}{|c|}{$\begin{array}{l}\text { No } \\
\text { cumplen }\end{array}$} & 1 \\
\hline \multicolumn{5}{|c|}{ Daños } & & & & \\
\hline & Sin daños & \multicolumn{3}{|c|}{1} & & & & \\
\hline & Ligero & \multicolumn{3}{|c|}{2} & & & & \\
\hline & Leve & \multicolumn{3}{|c|}{3} & & & & \\
\hline & Moderado & \multicolumn{3}{|c|}{4} & & & & \\
\hline & Extremo & \multicolumn{3}{|c|}{5} & & & & \\
\hline
\end{tabular}

variables de medición en la barra de menú como es el análisis estadístico descriptivo de los datos. Finalmente, la asignación de nuevos códigos arábigos para el ajuste óptimo en la obtención de un nuevo software de predicción de riesgo ecotoxicológico estuvo basada sobre la inquietud y preocupación por parte de la comunidad científica internacional sobre la contaminación de los ecosistemas acuáticos donde la valoración económica de los recursos involucrados es limitada.
(1) 


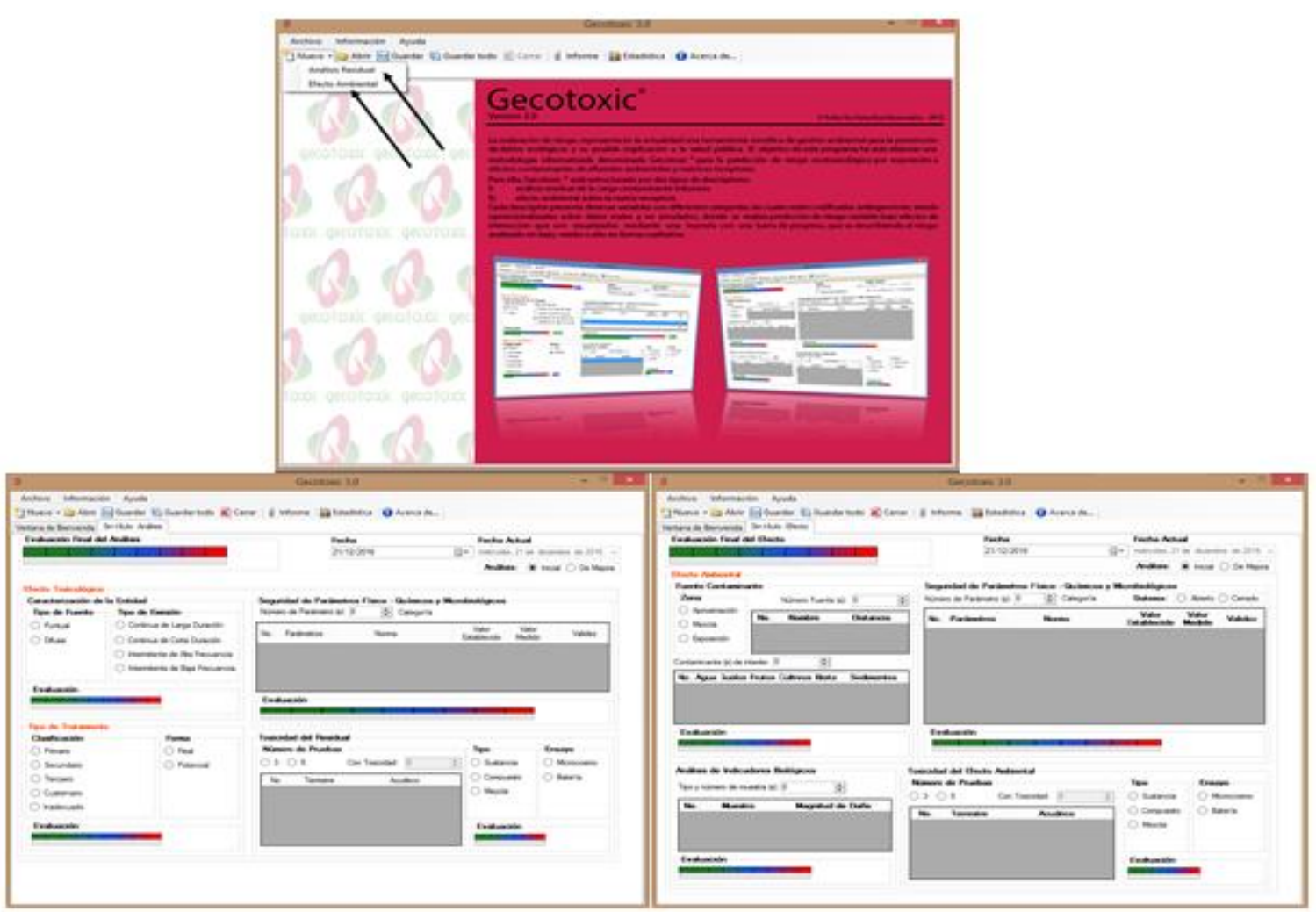

Figura 10. Ventana principal (superior), ventana de análisis residual (inferior izquierda) y ventana de efecto ambiental (inferior derecha) de GECOTOXIC.

En la optimización del riesgo ecotoxicológico, se consideró un intervalo más exacto para asignar puntuación sobre los parámetros físico-químicos que históricamente, han sido considerados para estimar la calidad ambiental de los ecosistemas acuáticos (Ramírez, León \& Piñeiro, 2008). Sin embargo, las fluctuaciones de los parámetros físico-químicos pueden ser tan normales que cualquier tipo de contaminación natural puede traer, en consecuencia, malas interpretaciones del comportamiento o salud ambiental de los ecosistemas (Lakshmanan et al., 2009).

De igual modo, se precisaron los códigos de puntuación sobre la magnitud del valor de daño en indicadores biológicos que pudieron ser los mismos en su condición de bioindicadores o biomonitores pensando en que los peces como organismos están considerados en el eslabón supe- rior de la cadena trófica de los ecosistemas acuáticos. Además, los peces representan son los primeros en ser utilizados para los protocolos de evaluación ecotoxicológica (Çiftçi, Ay, Karayakar, Cicik \& Erdem, 2015; Hamza, 2014; Heidary, Imanpour \& Monsefrad, 2011), conjuntamente en su condición de especies con categoría de centinelas para cualquier tipo de estudio ambiental (AbdAllah, 2017;Napán, Llanos, \& Paredes, 2010; Wilson, Figueiredo \& Machado, 2013;).

Cada nivel o diversos cambios biológicos que expresan los peces, sirven como señales de posible alteración ambiental de origen antropogénico y de esta forma, auxilia como indicador del riesgo ecotoxicológico a que una población natural puede estar expuesta, pues todo ente biológico tiene la capacidad potencial de ofrecer una respuesta ante cualquier estímu- 
lo que se presenta en el medio, pero cualquiera de la(s) respuesta(s) puede variar en forma correlacionada, si el organismo es considerado bioindicador o biomonitor, siendo esta última condición más reflexiva para "explicar" la calidad ambiental de los ecosistemas, ya que los biomonitores poseen mejores ventajas interpretativas (Cassanego, Sasamori, Petry \& Droste, 2015; Jebali et al., 2013;).

Asimismo, no solo es importante el uso de biomonitores como herramientas predictivas, ya que las nuevas tendencias, en la ecotoxicología acuática, están relacionadas con el uso de biomarcadores como expresión de síntesis sobre la información cualitativa y cuantitativa que describe la estimación del efecto para la salud antes de la exposición mediante la identificación de los dańos y la evaluación de dosis-respuesta, teniéndose en cuenta la información de los datos anteriores, disponibles en humanos, diferentes especies; y en sistemas in vitro cuando sea necesario para evaluar y regular los efectos de los contaminantes sobre las poblaciones en el tiempo (Wagner, 2002; Lock \& Bonventre, 2008; Planes \& Fuchs, 2015). En la nueva versión del software, el análisis de los biomarcadores es considerado dentro de la magnitud del valor de daño, siendo mejor interpretado para la predicción total del riesgo que se genera.

Se ha considerado que para la optimización del riesgo ecotoxicológico, lo que se concibe mediante el uso de otros modelos informatizados e índices ecológicos (Mirror, 2015; López, Figueroa, \& Corrales, 2016), así como mantener la realización de bioensayos, en condiciones controladas, donde el riesgo es igual al daño potencial por la exposición y ello, depende de la cuantificación con respecto al efecto toxicológico, expresión sobre la toxicidad formulada en términos de dosis, concentración y efecto letal para el 50\% de los individuos, valor máximo aceptable de concentración del tóxico, nivel más bajo de efecto observable (LOEC) y finalmente, el nivel más bajo de concentración no observable (NOEC) (Iannacone \& Alvariño, 2003; Pereyra, Rossini \& Darrigran, 2012; Sadeghi \& Hedayati, 2014).

Con el ajuste óptimo para la incertidumbre del nuevo software multifuncional para la predicción de riesgo ecotoxicológico, ya es pretendido lo que se desea, en la actualidad, y referido a la medición económica de la calidad ambiental, considerándose no solo el posible bienestar animal, sino además de todo recurso posible a medir (Butler, Corvalan \& Koren, 2005), haciendo conexión teórica entre los sistemas ecológicos y los económicos (Volk et al., 2007; Ryzyka, Strat \& Beijiang, 2014).

Se concluye que se establecieron los rangos de intervalos para máximos y mínimos, según progresión aritmética en el que se asignaron nuevos códigos arábigos de puntuación, ajustando el riesgo cualitativo según valores cuantitativos progresivos mediante análisis de similitud con daños, en un organismo biomonitor, existiendo mayor precisión y exactitud en el riesgo informatizado que se genera, para entenderse que cualquier tipo de estudio sobre los actuales problemas del entorno, ha motivado la construcción de un modelo que no solo explica, sino además, predice problemas al menos de un fragmento de la realidad relacionada con la contaminación ambiental sobre los ecosistemas acuáticos como se ha indicado (Blum, Galbraith, Henn \& Niss, 2007). 


\section{Referencias}

AbdAllah, A.T. (2017). Efficiency of invertebrate animals for risk assessment and biomonitoring of hazardous contaminants in aquatic ecosystem, a review and status report. Environ Risk Assess Remediat, 1(1), 22-24.

Argota, P.G. \& Iannacone, O.J. (2014). Metodología informatizada GECOTOX para la predicción de riesgo ecotoxicológico por exposición a efectos contaminantes en efluentes ambientales y ecosistemas acuáticos. Aceptado para su publicación. Rev. The Biologist, 12(2), 1-25.

Barbosa, J.C. (2003). What is mathematical modeling. En S. J. Lamon, W. A. Parder \& K. Houston (eds.), Mathematical modelling a way of life, Chichester, Ellis Horwood. Recuperado de: http://subs.emis. de/journals/ZDM/zdm063a8.pdf

Barbosa, J.C. (2006). Mathematical modelling in classroom: a critical and discursive perspective. $Z D M$, the International Journal on Mathematics Education, 38(3), 293-301.

Blum, W., Galbraith, P.L., Henn, H.W. \& Niss, M. (2007). Modelling and Applications in Mathematics Education. The 14th ICMI Study. New York, NY: Springer Science + Business Media, LLC.

Butler, C.D., Corvalan, C.F. \& Koren, H.S. (2005). Human health, well-being, and global ecological scenarios. Ecosystems, 8(2), 153 162. Recuperado de: https://doi. org/10.1007/s10021-004-0076-0
Camarena, G.P. (2000). Los modelos matemáticos como etapa de la matemática en el contexto de la ingeniería, reportedeinvestigación, México, ESIME-IPN. Recuperado de: http:// funes.uniandes.edu.co/6243/1/ CamarenaLamatem $\%$ C3\% A 1 ticaALME2003

Cassanego, M.B.B., Sasamori, M.H., Petry, C.T. \& Droste, A. (2015). Biomonitoring the genotoxic potential of the air on Tradescantia pallida var. purpurea under climatic conditions in the Sinos River basin, Rio Grande do Sul, Brazil. Brazilian Journal of Biology, 75(4), 79-87. Recuperado de: https://doi. org/10.1590/1519-6984.05514

Çiftçi, N., Ay, Ö., Karayakar, F., Cicik, B. \& Erdem, C. (2015). Effects of zinc and cadmium on condition factor, hepatosomatic and gonadosomatic index of Oreochromis niloticus. Fresenius Environmental Bulletin, 24(11A), 3871-3874.

Cordero, F., Suárez, L., Mena, J., Arrieta, J., Rodríguez, R., Romo, A.,..., Solis, M. (2009). La modelación y la tecnología en las prácticas de enseñanza de las matemáticas. Acta Latinoamericana de Matemática Educativa, 22(22), 1717-1726.

Hamza, C.A. (2014). Usefulness of Bioindicators and Biomarkers in Pollution Biomonitoring. International Journal of Biotechnology for Wellness Industries, 3, 19-26. Recuperado de: https://doi.org/10.6000/19273037.2014.03.01.4 
Heidary, S., Imanpour, N. \& Monsefrad, F. (2011). Bioaccumulation of heavy metals $\mathrm{Cu}, \mathrm{Zn}$, and $\mathrm{Hg}$ in muscles and liver of the stellate sturgeon (Acipenser stellatus) in the Caspian Sea and their correlation with growth parameters. Iranian Journal of Fisheries Sciences, 11(2), 325-337.

Iaanacone, J. \& Alvarińo, L. (2003). Efecto ecotoxicológico agudo del mercurio sobre Larvas del "Muy Muy" emerita analoga (Stimpson) (Decapoda: Hippidae) procedentes de cuatro localidades de Lima. Ecol. Apl., 2(1), 111-115.

Jebali, J., Khedher, S., Sabbagh, M., Kamel, N., Banni, M. \& Boussetta, H. (2013). Cholinesterase activity as biomarker of neurotoxicity: utility in the assessment of aquatic environment contamination. Revista de Gestão Costeira Integrada, 13(4), 525-537. Recuperado de: https:// doi.org/10.5894/rgci430

Lakshmanan, R., Kesavan, K., Vijayanand, P., Rajaram, V. \& Rajagopal, S. (2009). Heavy metals accumulation in five commercially important fishes of Parangipettai, Southeast coast of India. Advance Journal of Food Science and Technology, 1, 63-65.

Lehrer, R. \& Schauble, L. (2000). The development of model-based reasoning. Journal of Applied Developmental Psychology, 21(1), 39-48.

Lesh R. \& English L. (2005). Trends in the evolution of the Models and Modeling perspectives on mathematical learning and problem solv- ing. $Z D M$, the International Journal on Mathematics Education, 37(6), 487-489.

Lesh, R. \& Harel, G. (2003). Problem solving, modeling, and local conceptual development. Mathematical Thinking and Learning, 5(2), 157-189.

Lock, E.A. \& Bonventre, J.V. (2008). Biomarkers in translation; past, present and future. Toxicology,245(3), 163-166.Recuperadode: https://doi.org/10.1016/j. tox.2007.12.004

López, I. D., Figueroa, A. \& Corrales, J. C. (2016). Un mapeo sistemático sobre predicción de calidad del agua mediante técnicas de inteligencia computacional. A Systematic Mapping of Water Quality Prediction Using Computational Intelligence Techniques, 15(28), 35-51. Recuperado de: https://doi.org/10.22395/rium. v15n28a2

Mirror, P. (2015). Water quality index of estuarine environment. Current Science, 108(8), $1430-1433$.

Napán, K., Llanos, C. \& Paredes, C. (2010). Toxicidad aguda de metomilo en Poecilia latipinna (Lesueur, 1821) (Poecilidae). The Biologist, 8(1), 21-28. Recuperado de: http://sisbib.unmsm.edu.pe/ bvrevistas/biologist/v08_n1/pdf/ a02v8n1.pdf

Pereyra, P.J., Rossini, G.B. \& Darrigran, G. (2012). Toxicity of Neem's oil, a potential biocide against the invasive mussel Limnoperna fortunei (Dunker 1857). Anais Da 
Academia Brasileira de Ciencias, 84(4), 1065-1071. Recuperado de: https://doi.org/10.1590/S000137652012005000059

Planes, E. \& Fuchs, J. (2015). Cuáles son los aportes de la ecotoxicología a las regulaciones ambientales. Ciencia E Investigación, 65, 45-62. Recuperado de: http://aargentinapciencias.org/2/images/RevistasCeI/tomo65-2/5-Planes-cei65-2-5.pdf

Ramírez, M.A.P., León, M.M.L. \& Piñeiro, P.S. (2008). Metales pesados en trucha (Micropterus salmoides floridanus) de la presa Habanilla, Cuba. Revista AquaTIC, 29, 1-9.

Repetto, M. \& Sanz, P. (1995). Fundamentos de ecotoxicología. En M. Repetto (ed.), Toxicología Avanza$d a$, pp. 147 - 205. Madrid: Editorial Días de Santos.

Ryzyka, O., Strat, E.I. \& Beijiang, R. (2014). Ecological risk and economic loss estimation of heavy metals pollution in the Beijiang river, 21(2), 189-199. Recuperado de: https://doi.org/10.2478/eces2014-0015

Sadeghi, A. \& Hedayati, A. (2014). Investigation of LC50, NOEC and LOEC of glyphosate, deltamethrin and pretilachlor in guppies ( Poecilia Reticulata). Iranian Journal Of Toxicology, 8(26).

Schoenfeld, A.H. (2007). Problem solving in the United States, 1970-
2008: research and theory, practice and politics. $Z D M$, the International Journal on Mathematics Education, 39(5-6), 537-551.

Spahn, S.A. \& Sherry, T.W. (1999). Cadmium and lead exposure associated with reduced growth rates, poores fledging success of little blue heron chicks (Egretta caerulea) in south Lousiana Wetlands. Arch Environ Contam Toxicol, (37), 377-384.

Trigueros, M. (2006). Ideas acerca del movimiento del péndulo: un estudio desde una perspectiva de modelación. Revista Mexicana de Investigación Educativa, 11(31), 12071240.

Volk, M., Hirschfeld, J., Schmidt, G., Bohn, C., Dehnhardt, A., Liersch, S., \& Lymburner, L. (2007). A SDSS-based ecological-economic modelling approach for integrated river basin management on different scale levels - The project FLUMAGIS. Water Resources Management, 21(12), 20492061. Recuperado de: https://doi. org/10.1007/s11269-007-9158-z

Wagner, J.A. (2002). Overview of biomarkers and surrogate endpoints in drug development. Disease Markers, 18(2), 41-6. Recueprado de: https:// doi.org/10.1155/2002/929274

Wilson, G., Figueiredo, M. \& Machado, J. (2013). Dissipation and environmental risk of fipronil on aquatic environmental. The Biologist (Lima), 11(1), 107-117. 